This is an Authors' Accepted Manuscript of an article published in SEXUALITY \& CULTURE, Online First, copyright Springer, available at: https://link.springer.com/article/10.1007/s12119-017-9434$\underline{x ? w t \_m c=\text { Internal.Event.1.SEM.ArticleAuthorOnlineFirst }}$

\title{
Between Homohysteria and Inclusivity: Tolerance towards Sexual Diversity in Sport
}

\author{
Joaquín Piedra \\ Department of Physical Education and Sports, Universidad de Sevilla, Spain \\ Rafael García-Pérez \\ Department of Educational Research Methods and Diagnostics, Universidad de Sevilla, Spain \\ Alex Channon \\ School of Sport and Service Management, University of Brighton, UK
}

Corresponding Author:

Dr. Joaquín Piedra, Departament of Physical Education and Sports, Universidad de Sevilla, C/ Pirotecnia s/n, 41013 Seville, Spain

Email: jpiedra@us.es 


\title{
Between Homohysteria and Inclusivity: Tolerance towards Sexual Diversity in Sport
}

\author{
Joaquín Piedra \\ Department of Physical Education and Sports, Universidad de Sevilla, Spain \\ Rafael García-Pérez \\ Department of Educational Research Methods and Diagnostics, Universidad de Sevilla, Spain
}

\author{
Alex Channon \\ School of Sport and Service Management, University of Brighton, UK
}

\begin{abstract}
The small number of openly gay or lesbian people in top-flight sport raises the question about whether sporting or social contexts makes it easy or difficult for these sportspeople to come out. There are studies conducted in the UK and USA using the theory of homohysteria but the scarcity of studies in Spain on this subject, added to the lack of a tool with which to measure tolerance towards sexual diversity in sport, has led us to write this paper in which we analyze the suitability of a new tool for classifying the metacognitive profiles of two samples from different cultures, relative to the concept of homohysteria. This correlational study involved 879 men and women aged 16-78 $(\mathrm{M}=28.45$ years; $\mathrm{SD}=13.08)$ living in either Spain or the United Kingdom, who were actively participating in or following sport of some kind. Results show that the new instrument has adequate construct validity and high reliability $($ Alpha $=.95)$, facilitating the measurement of two dimensions of tolerance towards non-heteronormative sexualities in sport: non-rejection and acceptance. Results point to three metacognitive profiles in terms of fit of the level of tolerance in these two dimensions: low, high, and partial. Findings show high levels of both dimensions of tolerance in the UK, and higher percentages of partial tolerance in Spain, underlining the importance of cultural contexts and policies which may affect people's levels of tolerance. We conclude that a pseudo-inclusive climate exists in Spanish society today.
\end{abstract}

Keywords: Homophobia; Sexual attitudes; Culture; Gender 


\section{Introduction: Sport, Gender and Homohysteria}

Historically, sport has been an area of male dominance, and specifically a site for the maintenance of hegemonic masculinity (Connell 1995), where gender and sexual diversity has been obscured, and sexual minority individuals have been stigmatized or repudiated (Davis-Delano 2014; O’Brien, Shovelton and Latner, 2013; Pronger 2000). Research has identified a wide range of types of discrimination (Barber and Krane 2007), with people not fitting these stereotypes being forced to remain invisible and silent under the weight of heteronormativity and outright discrimination (Krane and Barber 2003).

However, given that cultures change and evolve, more recent studies have revealed shifting attitudes in sport towards sexual diversity and sexual minorities. For instance, the research by Kauer and Krane (2006) highlights that the "coming-out" of women in a women's team had a positive effect on the attitudes of heterosexual players. The longitudinal study involving rugby and hockey players in the UK by Anderson, McCormack and Lee (2012) highlights the reduction in recent years of negative attitudes towards homosexuality among sportspeople, with certain homosexualized behaviors which were hitherto singled out and rejected by men now being accepted. In a similar vein, the studies by Ensign, Yiamouyiannis, White and Ridpath (2011) and Oswalt and Vargas (2013) find positive attitudes towards Lesbian, Gay, Bisexual and Transgender (LGBT) athletes, with there being only $15 \%$ of male and female trainers in their sample who demonstrated negative attitudes. Additionally, similar changes are evident in sport media coverage. Here, Channon and Matthews (2015) found inclusive and accepting discourse in online media coverage of an American mixed martial arts fighter who had a history of performing in gay pornography, while Cleland (2015) found users of UK-based, football-themed Internet forums actively challenging homophobia and espousing inclusion towards gay footballers. It appears that these more accepting climates within sport become stronger when direct contact is made with LGBT people (Adams \& Anderson, 2012; Anderson, Magrath and Bullingham, 2016; Ensign et al. 2011).

In understanding the complexity of such changes, and to help theorize the dynamics through which shifting levels of homophobia impact on climates of hostility and acceptance within sport, Eric Anderson (2009) proposed the concept of homohysteria within his broader theory of inclusive masculinity (Anderson, 2009; 2014; Anderson \& McCormack, 2016). Defining this as "the fear of being socially perceived as gay" (Anderson \& McCormack, 2016, p.2), along with engaging in actions intended to distance oneself from the suspicion of being gay, Anderson notes that among men, homohysteria is typically manifested by fleeing from feminized behavior, including physical contact with other men or showing signs of affection and emotion, while also 
maintaining homophobic discourse. Homohysteria helps to explain how gendered patterns of behavior - both within and outside of sport settings - play out in relation to shifting levels of societal homophobia, particularly highlighting how homophobia can affect the behavior of individuals who may not necessarily be homophobic themselves (McCormack \& Anderson, 2014). It thereby adds an important dimension to analyses of homophobia, inclusion, and social change by identifying the impact homophobia has on wider performances of gender and the construction of normative climates pertaining to the acceptance (or not) of sexual diversity.

Anderson et al. (2016) propose that, driven by social, cultural and legislative processes, levels of homohysteria and homophobia in society may change over time, such that societies may move through three possible stages with respect to these interrelated phenomena:

1) Homoerasure, featuring societies with high levels of homophobia and heteronormativity, within which homosexuality is not recognized as a stable or coherent sexual identity. Here, men can engage in otherwise feminized behaviors without being singled out as potentially gay, because there is no assumed link between the two given the widespread erasure of homosexuality, and thus, very little homohysteria;

2) Homohysteria, featuring societies with a generalized homophobic culture where diversity is rejected and heteronormative masculinity and femininity are emphasized, particularly as a means of proving one's heterosexuality. In this setting, signs of intimacy or emotions between people of the same sex - or indeed, any gender transgressive behavior at all - is problematic as it invites suspicion of homosexuality;

3) Inclusivity, which includes societies where even though a certain level of homophobia still persists in places, in general positive attitudes prevail. There is greater liberty with respect to both the rights and social status of sexual minorities, but also the gendered behavior of all people given that being homosexualized is not considered so highly problematic. In other words, as homophobia decreases, homohysteria is diminished, and the acceptance of gender and sexual minorities becomes normalized.

Societies such as those in the UK and the USA, where studies have recently questioned the persistence of negative attitudes towards sexual minorities, are shifting into the third of these stages, becoming generally (although not universally) more accepting of sexual and gender diversity (Anderson \& McCormack, 2016). Importantly, even within the traditionally gender-conservative cultural sphere of sport, research has confirmed 
these trends, indicating that inclusive attitudes have permeated across even some of the most historically homophobic spaces of these societies (Anderson et al. 2016).

However, in other, non-English speaking countries, a lack of studies in sport does not allow an accurate diagnosis of the situation to be made. In Spain, as Barbero (2003) points out, sport researchers have not taken much interest in the question of sexual diversity. We have had to wait for more than a decade since that observation was made for the first studies in this area. In a study with undergraduate students, Piedra (2015) shows a wide range of possibilities in Spain's sporting context, from blunt rejection to acceptance of gays and lesbians. This study underlines the controversy that sexuality generates among sportspeople, particularly in team sports, with a climate of rejection of gays and lesbians often persisting. It highlights that Spanish society trails behind others where there is a climate of greater tolerance. In turn, the students explain that people find it difficult to assimilate social changes that are occurring relatively quickly, which is why the first impressions in these situations are of rejection or lack of tolerance. This first stage would be overcome, according to them, once these first moments have been negotiated. However, this change in Spain does not necessarily mean greater levels of acceptance; rather, a certain degree of respect is shown which may be largely due to a fear of being labeled homophobic (see McCormack and Anderson 2010). As such, the students in Piedra's (2015) study argue that Spanish people frequently adopt "politically correct" positions regarding gays and lesbians, while concealing their true attitudes and opinions.

Obviously, it is not possible to access this knowledge and diagnose this imbalance between social positioning and genuine attitude from a traditional cognitive-affective perspective. However, the metacognitive social approach (Chin and Kuo 2010) makes it possible to explore the assessment of tolerance and distinguish the executive control or cognitive style which fits the adopted social position, considering the degree of tolerance within a continuum between "non-rejection" and "acceptance". This perspective in the measurement of tolerance opens its own field of reflection about its measurement in the world and culture of sport, and it also constitutes a new theoretical base for the construction of a measure of tolerance capable of discriminating between tolerance (or not) executed mentally in a profound and adjusted way, as opposed to the production of a "politically correct" style of thinking which is essentially maladjusted, limited by its superficiality and with little possibility of real application. 


\section{Diagnosis of Tolerance in Sport}

The scale developed by Herek (1984), Attitudes towards Lesbians and Gays (ATLG), has often been used in research to measure people's attitudes. It is a 20 item Likert-type scale with five response options. It is divided into two subscales each with 10 items, referring to Attitudes towards Lesbians (ATL) and Attitudes towards Gays (ATG) respectively. The reliability and validity of the tool has been demonstrated (Herek 1988), showing adequate internal consistency $(a l p h a>.85)$. The instrument has been applied and validated in Spanish (Herek and González-Rivera 2006), although it should be noted that this work was conducted among Hispanic social groups in the United States. Some of the previously mentioned studies in the area of sport applied this scale to gauge the attitudes of certain groups (Ensign et al. 2011; O’Brien et al. 2013; Oswalt and Vargas 2013) with a wide range of findings. Furthermore, this tool was not designed specifically for the area of sport, so it does not take into account the historical and cultural peculiarities of sports culture, which - as noted at the outset - have traditionally reproduced heterosexism and homophobia. Recently, Piedra (2016) has published the Attitude Scale towards Sexual Diversity in Sport. This new tool, an 18-item Likert-type scale, has been applied and validated in Spain and presents adequate internal consistency (alpha $>.90)$, although the instrument was not specifically developed with a view to accounting for cultural differences between British and Spanish societies, reducing its utility in the context of the current study.

There are other questionnaires for specific contexts of physical exercise such as physical education (PE) classes, with two scales (Perceptions of Homophobia and Heterosexism in Physical Education-PHHPE) measuring the perception of homophobia and heterosexism (Morrow and Gill 2003) amongst both teachers (PHHPE-TS) and students (PHHPE-SS). These tools have been applied subsequently in different studies, both in English-speaking countries (Gill, Morrow, Collins, Lucey and Scultz 2010) and in Spain after translation and adaptation (Piedra, Rodríguez-Sánchez, Ries and Ramírez-Macías 2013). However, these scales do not measure individuals' own attitudes as such, and they do not include a psychometric study to ensure validity and reliability, while their scope is limited to the educational application of sport rather than competitive contexts. Further, these scales only measure perceptions regarding lesbians and gays. It would be interesting to have a tool to cater for other minority gender identities and sexual orientations as well, such as transgender people, because as Caudwell (2014) and Griffin (2012) point out, the lack of recognition and visibility towards this group in particular serves only to increase negative attitudes and feelings towards them. In sport, such erasure from discourse is often translated into harmful stereotyping, rejection, and disdain towards trans athletes. 
Another aspect to consider is the nature of the object of study and the focus on the social formation of the ideas and attitudes we handle. In this sense, from the approach of social constructivism, the metacognitive focus (Chin and Kuo 2010; Martí 1995) makes it possible to observe the capacity for planning and decisionmaking in relational settings, specifically, in relation to the assessment of degrees of tolerance towards sexual diversity in the world of competitive sport. In this sense, the plane of social metacognitive regulation, in relational decision-making (on inclusion of sexual and gender diversity), is exercised from a continuum established between the "non-rejection" and "acceptance" of sexual diversity.

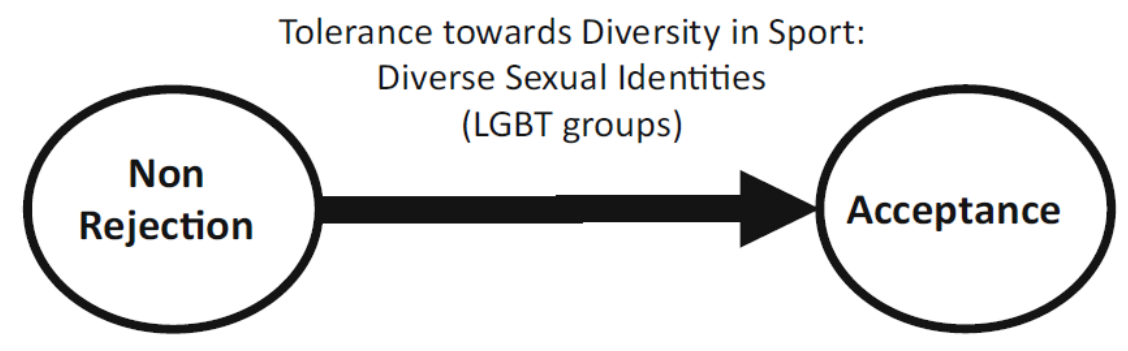

Fig. 1 Non rejection-acceptance continuum of sexual diversity in sport

This continuum effectively broadens the horizon between the stages of "homohysteria" and "inclusion" described by Anderson (2009), by attempting to analyze the possibility that professed acceptance of sexual diversity may take different forms. Specifically, these involve either avoiding or stigmatizing outright, homophobic discrimination ("non-rejection"), or advocating the viewpoint that non-heteronormative sexualities are legitimate, socially valuable, and worthy of celebration (“acceptance"). While this continuum model may imply direct continuity or perhaps a causal, developmental relationship between the two forms, from the point of view of Piedra's (2015) sample of students, a professed tolerance which conforms to standards of political correctness by simply avoiding homophobia may not, in fact, correspond at all with acceptance.

In this sense, we propose the possibility of identifying an intermediate state of tolerance of sexual diversity, between the end of the stage of homohysteria and the initiation of the stage of social inclusivity of diversity as outlined above. This intermediate state corresponds to certain premises involved at the end of the previous stage, such as maintaining a politically correct discourse and the non-rejection of potentially homosexualizing behaviors. However, the general feature remains of the high social value attributed to heteronormative gender performance, particularly within the traditionally gender-conservative field of 
competitive sport and, in short, the incomplete acceptance of inclusive discourses and principles. As Piedra (2015) points out, "change is not always accompanied by higher levels of acceptance, but simply a certain respect perhaps through fear of being singled out as homophobic" (p. 1072), echoing the notion that homophobia, and not homosexuality, is now stigmatized (McCormack and Anderson 2010). For that reason, we refer to this stage as pseudo-inclusivity, where individuals within a society adopt discourses and maintain preferred appearances related to the inclusion of diversity, but fall short of the characteristics attributed to Anderson and colleagues' (2016) inclusive stage, particularly with respect to the acceptance that a variety of sexual and gender identities are legitimate and valuable. However, none of the aforementioned scales offer the possibility of measuring this phenomenon.

With this in mind, the overall objective of the present study was therefore to elaborate a diagnostic procedure to enable the differentiation of the "pseudo-inclusive" stage and permit the assessment of differences in development between English-speaking (United Kingdom) and Hispanic (Spain) cultures, and in so doing make a contribution to the diagnosis of tolerance in sport. More specifically, our research objective was to draw up a scale to measure the levels of tolerance towards various aspects of sexual diversity in sport. Our study included the following specific objectives:

1) Describe the level of tolerance towards sexual diversity in sport for the overall sample obtained in Spain and the UK. This meant constructing a specific tool to measure and validate sportspeople's attitudes to assess their metacognitive adjustment in tolerance in two dimensions:

a) Dimension one, measuring the level of "non-rejection" regarding the participation of LGBT people in sport; and, directly related to this;

b) Dimension two, measuring their level of "acceptance".

2) Identify metacognitive profiles as a function of both dimensions of the measure of attitudes of tolerance towards sexual diversity in sport.

3) Characterize these profiles and contrast the samples from both countries, identifying and theorizing the main differences.

\section{Material and methods}

This research adopted an ex post facto methodology, adopting a correlational design through (Likert scale) self-report questionnaires, considering contrast as a function of the cultural origin of data (UK/Spain). With this design we did not mean to modify the variables which were the object of study, but explore their 
nature and behaviour in the samples analyzed and assess the differences which could be associated to each of the cultural contexts compared.

\section{Participants}

The study involved 879 participants (69.5\% male and 30.5\% female), aged between 16 and 78 years (M $=28.45$ years $; \mathrm{SD}=13.08)$. Of these, $67.7 \%$ performed sport within a federation, while $32.2 \%$ did so outside a federative structure. Similarly, seven out of ten participants were involved in team sports, while the others performed within individual disciplines. In terms of nationality, $56.2 \%$ were Spanish, $34.5 \%$ British, with the remaining $9.7 \%$ of nationalities coming from the rest of the world, although these participants were all living in either Spain $(1.6 \%)$ or the UK $(8.1 \%)$ at the time of the study. A majority of them $(75.3 \%)$ had higher education backgrounds (further education, college, under- or postgraduate). Similarly, a majority of the participants $(88.9 \%)$ stated that they were heterosexual, while the remaining percentage was divided among different sexual orientations: $3.2 \%$ identified as lesbians, $2.3 \%$ as gays, $2.5 \%$ as bisexuals, and $0.6 \%$ as others.

\section{Instrument}

The questionnaire collected information about participants' sociodemographic characteristics: gender, age, sport (group or individual), and membership of sports associations; and sample background information: the country in which they completed the questionnaire, and their country of origin. It also included a Likert-type scale with 32 items grouped into two dimensions to measure tolerance towards sexual diversity from two perspectives: 1) the dimension of "non-rejection" of sexual diversity in the world of sport (12 items); and, 2) the dimension of "acceptance" of harmonious coexistence with this sexual diversity in the practice of sport (20 items). Here, the "non-rejection" questions centered on more abstract discussion around the visibility of both LGBT people within sport and non-heteronormative behaviors among athletes, as well as ideas about the importance of 'equality' in sport. Meanwhile, the "acceptance" questions were more directly related to participants' feelings, particularly with respect to experiences of coming into close proximity with LGBT people themselves, and their personal interpretation of others' gendered behavior. The Likert-type response scale had 5 points (from 1 -totally disagree- to 5 -totally agree-). The reliability study applied using optimal scaling $(\mathrm{CATPCA})$ gave a high overall coefficient (Cronbach's Alpha $=.95)$; with the independent variables for each dimension also being high (Alpha D1 = .97; Alpha D2 =.88), demonstrating that reliable measures were taken.

In turn, on the basis of the validity of content and association of the different items for the measurement of each of the two dimensions $(\mathrm{D} 1=$ non rejection; $\mathrm{D} 2=$ acceptance $)$ construct validity was demonstrated by 
applying exploratory factorial analysis with the Main Component (MC) method and Oblimin Rotation (Table 1), finding the hypothesized bifactorial structure by obtaining high rates of mean saturation of the items in its factor $\left(\mathrm{M}_{\mathrm{f} 1}=.796, \mathrm{DT}_{\mathrm{f} 1}=.076 ; \mathrm{M}_{\mathrm{f} 2}=.617, \mathrm{DT}_{\mathrm{f} 2}=.104\right)$, revealing a valid measurement for the main variables. The new variables were calculated by adding the Likert-type items for each dimension (20 acceptance items which configured a subscale from 20 to 100 points and 12 items of non-rejection which configured another subscale from 12 to 60 points). However, to facilitate comprehension this was divided by the number of items in each factor in order to maintain the measure on the same original 1-5 point scale.

Table 1.

Factorial structure of scale on tolerance in sport. Own source.

Configuration matrix ${ }^{1}$ : saturation of items in each dimension of tolerance

\begin{tabular}{|c|c|c|}
\hline \multirow[b]{2}{*}{ Items ${ }^{2}$ for measuring tolerance towards sexual diversity in sport } & \multicolumn{2}{|l|}{ Components } \\
\hline & $\begin{array}{l}1 \\
\text { Acceptance }\end{array}$ & $\begin{array}{l}2 \\
\text { Rejection }\end{array}$ \\
\hline $\begin{array}{l}\text { i28. I would never be part of a sports club that included homosexual, bisexual or } \\
\text { transsexual people }\end{array}$ & .907 & \\
\hline $\begin{array}{l}\text { i32. If I were a coach, I would not feel comfortable knowing that there is a } \\
\text { homosexual person on my team }\end{array}$ & .898 & \\
\hline i21. I wouldn't like my child's coach to be gay or lesbian & .874 & \\
\hline i31. I would not feel comfortable hugging a homosexual rival after a match & .862 & \\
\hline i25. I would not feel at ease if it is known that my teammate is not heterosexual & .862 & \\
\hline $\begin{array}{l}\text { i29. I would not like to get changed in the same changing room with a homosexual } \\
\text { person }\end{array}$ & .858 & \\
\hline $\begin{array}{l}\text { i14. I think that boys are not genetically suited for 'artistic' sports such as figure } \\
\text { skating, rhythmic gymnastics or aerobics }\end{array}$ & .838 & \\
\hline $\begin{array}{l}\text { i26. If I had a son, I would not feel at ease if he wanted to practice rhythmic } \\
\text { gymnastics or any other mostly 'feminine' sports }\end{array}$ & .812 & \\
\hline i30. Girls who practice contact sports, like rugby, lose part of their femininity & .812 & \\
\hline $\begin{array}{l}\text { i3. If a sportsman touches a teammate's bottom when scoring a goal, it is because } \\
\text { he is gay }\end{array}$ & .798 & \\
\hline i18. If I had a daughter, I would not feel comfortable if she competed in rugby & .795 & \\
\hline i10. Generally, I think that girls who practice sport are too muscular & .789 & \\
\hline $\begin{array}{l}\text { i20. I think that lesbians are more aggressive in sport than heterosexual women and } \\
\text { girls }\end{array}$ & .788 & \\
\hline $\begin{array}{l}\text { i7. It seems logical to me that sports fans would laugh at an effeminate player during } \\
\text { a game }\end{array}$ & .785 & \\
\hline $\begin{array}{l}\text { i17. I would not like to get changed in the same changing room with a transsexual } \\
\text { person }\end{array}$ & .764 & \\
\hline i8. I would feel nervous in an openly homosexual sports club & .757 & \\
\hline i16. If I had children, I would not like their coach to be transsexual & .749 & \\
\hline i22. I think that most girls who practise my sport are lesbians & .730 & \\
\hline $\begin{array}{l}\text { i1. The image of lesbian sportswomen kissing each other to celebrate a victory } \\
\text { should be avoided in sport events }\end{array}$ & .633 & \\
\hline $\begin{array}{l}\text { i19. Gay sportspeople should not kiss each other and show off their homosexuality } \\
\text { within public sporting events }\end{array}$ & .626 & \\
\hline
\end{tabular}


i27. If I had children, I would be equally glad if they had either a heterosexual or homosexual coach

i24. I think it is fine to participate in sporting competitions with openly homosexual people

i15. I would feel at ease in my team with a homosexual teammate

i12. I would not mind people I take part in a homosexual, bisexual or transsexual sports club

i5. I would feel at ease in my team with a transsexual teammate

i13. A girl can remain feminine if she practices a combat sport, like boxing

i23. I would not like to hear the word 'faggot' used as an insult in stadiums

i9. If I had a child, I would enjoy watching them practicing rhythmic gymnastics

i4. I don't mind people watching me shaking my hand with a homosexual rival after a match

i6. I would not like to hear the word 'dyke' used as an insult in stadiums

i2. If I had a daughter, I would be glad to hear that she wanted to practice rugby i11. I would feel at ease knowing that my coach is not heterosexual

1. Extraction: analysis of main components. Rotation: Oblimin normalization with Kaiser.

2. Items are presented ordered according to their factorial weight in the dimensions, weights below .350 have been eliminated

Finally, we calculated the overall mean of tolerance towards sexual diversity in sport with the sum of the entire scale (32 items). This scale consists of 32 to 160 points, and it serves as a conceptual benchmark for establishing groups among participants depending on whether they present low (32-64), average (65-96), or high (97-160) tolerance. Although these benchmarks are of course arbitrary, they nevertheless serve as a straightforward means of comparing profiles of answers, derived from the 1-5 scaling our participants used themselves.

\section{Procedure}

Participants were gathered in two ways, depending on the country. In Spain, they completed the questionnaires in training sessions or before competition, with the consent of the clubs. The questionnaire took 15 minutes to complete in this format. A telematic procedure was adopted for the British part of the sample. We emailed the presidents or secretaries of different clubs covering a variety of sports around Britain. We informed them about the project and asked them to distribute the scale online to its athletes. The scale was also posted on different websites for followers of clubs or sports whose members were invited to fill in the questionnaire.

Finally, the instrument was also distributed on the social networks of sports clubs. Participation was anonymous as the questionnaire was completed online, and all people over 16 who either practiced or followed sport were encouraged to take part. Participants completed the questionnaire voluntarily using their chosen electronic 
device, and they were free to choose whether to complete all or just part of it. It took about 10 minutes to complete the whole questionnaire in this manner.

\section{Data analysis}

We applied the SPSS package (version 23.0) for both the descriptive and correlational study of the items and for the analysis of validity and reliability using optimal scaling (CATPCA) and factorial analysis (PCOblimin). We also applied partition methods ( $k$-means clustering) to identify metacognitive profiles according to their level of adjustment to the "non-rejection - acceptance" continuum. We calculated the scores on each dimension or subscale and went on to study the distributions applying the Kolmogorov-Smirnov normality test, obtaining values for $p=.000$ in both dimensions (and for the overall score) and therefore showing their free distribution. This meant contrasting them with non-parametric techniques for related samples (Wilcoxon $z$ score) to compare the distributions between the two dimensions, and other contrasts between independent measures (Mann-Whitney $U$ test). Finally, we conducted a comparative/exploratory analysis for both countries (UK/Spain) as a function of sex, which involved the application of contingency tables and symmetry statistics (chi-square, Phi and CC).

\section{Results}

This section presents the results of the study. Firstly, we show the levels of tolerance of participants, with distributions according to the different dimensions of the scale, and the relations between them. Secondly, we identify the metacognitive profiles that stem from the results, highlighting their distribution as a function of the cultural contexts analyzed.

\section{Levels of Tolerance (Non-Rejection/Acceptance) of Sexual Diversity in Sport}

We begin with a general outline of the levels of tolerance towards diversity in sport from the overall score in tolerance, without distinguishing between non-rejection and acceptance. We will then go on to present the values obtained in each of the two dimensions and explain how the measures are differentiated according to the cultural and social setting. 


\section{Overall Tolerance Towards Sexual Diversity in Sport: Spain and UK}

Globally, when we valued tolerance in the total of the scale (32-160 points) without differentiating according to sample or dimensions, we obtained a mean of 108.35 points (median 96) and a high standard deviation of 29.107, presenting us with an open and complex distribution as shown below.
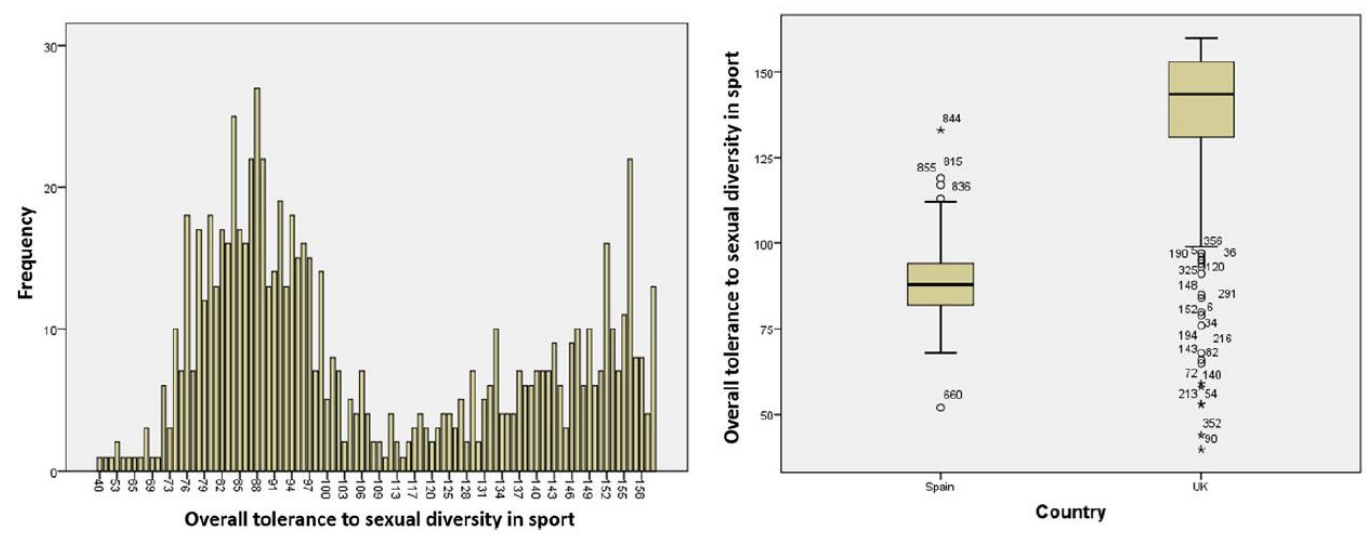

Fig. 2 Levels of tolerance towards sexual diversity in sport

In fact, observation of the first image led us to think that there were different sample subgroups which produced extreme internal variation. This was confirmed immediately when we looked at the significant differences in tolerance according to countries ( $U$ Mann-Whitney $=6946.500 ; p=.000)$, between the UK $(M=$ 137.88; $\mathrm{SD}=22.014)$ and Spain $(\mathrm{M}=88.11 ; \mathrm{SD}=9.366)$. This implied that the origin of the sample referred to a cultural context which had a significant impact on the level of overall tolerance. Specifically, for this contrast the size effect gave a Cohen's $d$ of 2.9 with $\mathrm{r}=.827$, which represented an appraisable impact.

It can be said with confidence that both contexts (countries) present tolerant attitudes towards sexual diversity but with clearly differing profiles, making it possible to identify the UK as significantly more tolerant and, therefore, following our initial hypothesis, it is reasonable to conclude from the data that this country can generally be described as being in the stage of inclusivity. In contrast, the Spanish sample obtained an average score which, according to the threshold indicated for the overall score of tolerance, did not reach similarly high levels as those we might expect of an inclusive society. However, a deeper analysis of the results reveals that this does not mean Spain is anchored in the rejection of people who manifest a diversity of sexual orientations. 


\section{Distributions of the Dimensions of Non-Rejection and Acceptance of Diversity}

Let us now consider the 1-5 scale which measured both dimensions of tolerance: 1) "non-rejection" obtained a mean of $3.95(\mathrm{SD}=0.750)$, which was a very high score; in turn, 2) "acceptance" obtained a mean score of $3.05(\mathrm{SD}=1.254)$, implying a mid-level of tolerance. The distributions are shown below:
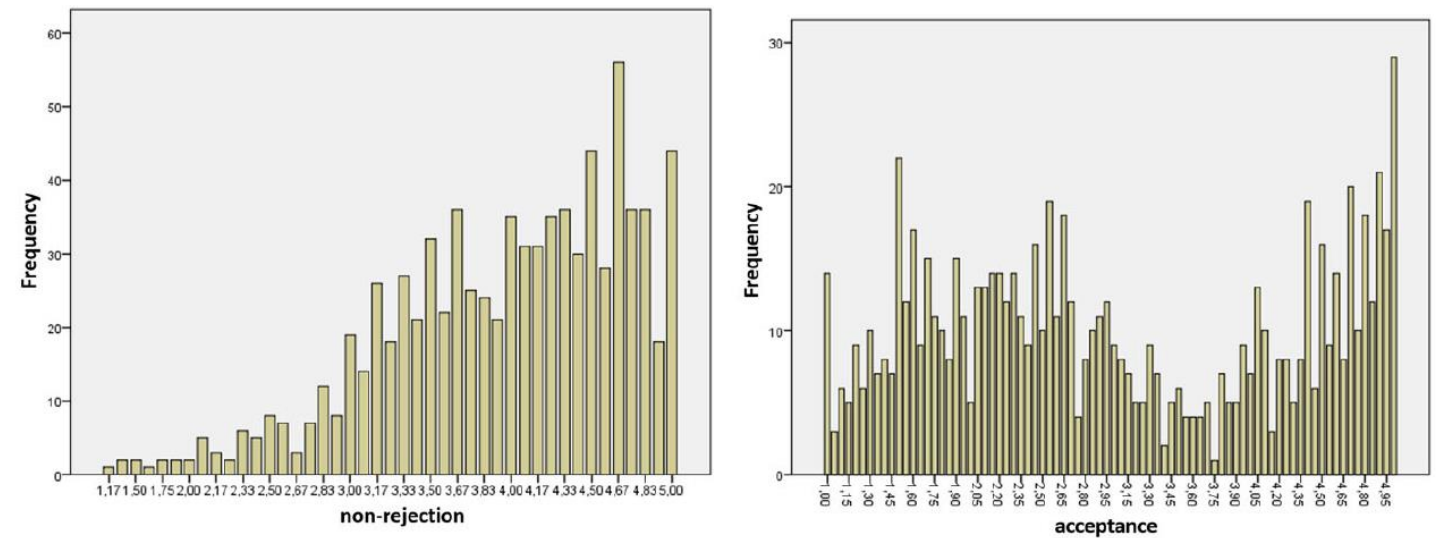

Fig. 3 Distributions of the dimensions of non-rejection and acceptance of diversity across the combined sample

As one can see, the effect of the coexistence of two significantly different sub-samples in the data can again be seen in the acceptance dimension; however, it is an effect which was not shown in non-rejection. Therefore, the acceptance variable is the one which had the most impact on the observable variability of tolerance. These results could lead us to think that both measures constituted more or less independent variables; an aspect which is dealt with in the following section.

\section{Relation and Independence of "Non-Rejection" vs. "Acceptance" of Sexual Diversity}

Analyzing the relation and independence of both distributions we obtained an ordinal correlation coefficient Rho $=.301(\mathrm{p}=.000)$, implying that the combined covariance of the two dimensions established a significant covariance. However, this result was obtained considering all the data from the study, but when we studied the relation between the two dimensions specifically for each country we found very pronounced differences in the form of an inverted relation (Figure 4). 

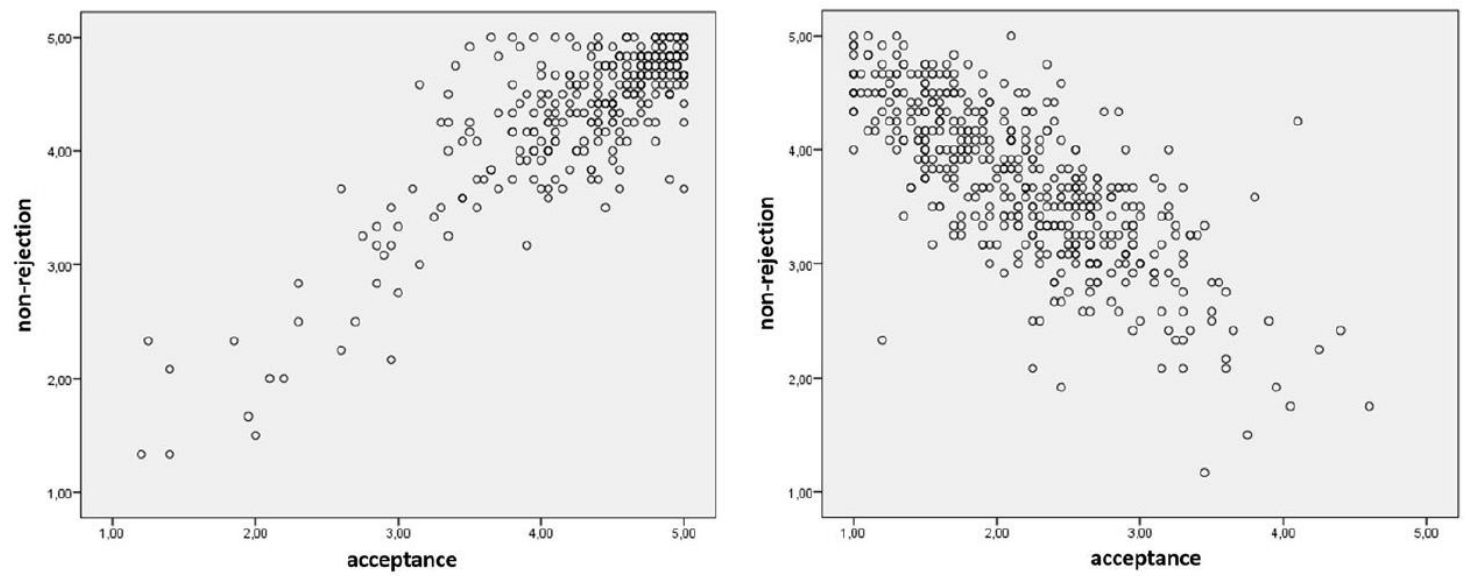

Fig. 4 Scatter plot between the dimensions "non-rejection/acceptance" comparing the data for the UK and Spain

While in the UK the correlation remained positive and high $(\mathrm{Rho}=.797 ; \mathrm{p}=.000)$, in Spain this relation was inverted, correlating less and negatively $(\mathrm{Rho}=-.418 ; \mathrm{p}=.000)$.

That makes clear that both variables covariate together, but in a different way under specific cultural circumstances. This can logically only happen if both distributions function independently. We checked this last aspect, formulating a hypothesis of independence which we studied with non-parametric statistics. The result obtained from Wilcoxon's non-parametric contrast test for two related samples $(Z=-16.908 ; p=.000)$ allowed us to reject the null hypothesis $\left(\mathrm{H}_{0}=\right.$ the two distributions were the same or very similar, indicating that they were two measures of the same feature). In short, although certain levels of covariance of the two dimensions were appreciated, it also showed that they constituted independent measures of two different features, expressed differently within the cultural context of Spain and the UK.

\section{Identification of Metacognitive Profiles Referring to Tolerance Towards Sexual Diversity in Sport}

A profound analysis of the problem presented here requires the study of both subsamples (UK/Spain) from the measures obtained in the study of both attitudinal features (non-rejection/acceptance) towards sexual diversity in sport. For that reason, we explored the formation of empirical groups with correlational procedures of multivariate classification (cluster analysis). The study of these profiles and their level of association to the two countries of origin allowed us to interpret the type of effects involved in the differentiation of these profiles of tolerance towards sexual diversity. 


\section{Profiles of Tolerance Towards Sexual Diversity in Sport}

The application of the (K-means) partition procedure provided us with a perspective enabling us to complete the viewing of results. We identified three well-differentiated groups with cluster centers (mean values) which situated each person according to: 1) their low tolerance; 2) their high tolerance; or 3) what we have called partial tolerance. This partition in three groups was seen as valid and explanatory of the differences and impacts which we studied on attitudes of tolerance towards sexual diversity in sport. Table 2 shows the results and the validity check of the grouping.

Table 2

Result of the (K-means) partition in 3 groups, exploratory ANOVA and impact of each factor. Own source.

\begin{tabular}{clc} 
Partition & ANOVA & Impact \\
GROUPS & Conglomerate Error & \\
\cline { 2 - 3 }
\end{tabular}

effect

\begin{tabular}{|c|c|c|c|c|c|c|c|c|c|c|}
\hline $\begin{array}{l}\text { Tolerance } \\
\text { factors }\end{array}$ & 1 & 2 & 3 & $\begin{array}{l}\text { Quadratic } \\
\text { mean }\end{array}$ & Gl & $\begin{array}{l}\text { Quadratic } \\
\text { mean }\end{array}$ & Gl & $\mathrm{F}$ & Sig. & $\begin{array}{l}\mathrm{d}_{\text {Cohen }} \\
\mathrm{r}\end{array}$ \\
\hline $\begin{array}{l}\text { F1. Non- } \\
\text { rejection }\end{array}$ & 3.11 & 4.54 & 4.20 & 143.601 & 2 & .189 & 764 & 760.183 & .000 & $\begin{array}{l}3.1 \\
.843\end{array}$ \\
\hline $\begin{array}{l}\text { F2. } \\
\text { Acceptance }\end{array}$ & 2.74 & 4.50 & 1.68 & 524.891 & 2 & .200 & 764 & 2624.841 & .000 & $\begin{array}{l}7.0 \\
.961\end{array}$ \\
\hline
\end{tabular}

Three groups were confirmed, following the above-mentioned pattern, with the first two being for low and high scores (correlating highly between them) pointing to two groups with a clear metacognitive adjustment in the "non-rejection and acceptance" continuum. The first group rejected and did not accept, which we could basically classify as "intolerant" (low tolerance). The second group did not reject and accepted sexual diversity in sport; it constituted the "tolerant" group (high tolerance). However, the third group did not reject, but nor did it accept, pointing to an important cognitive maladjustment which was shown even in the presence of inverse correlations between these two dimensions.

In other words, we detected a large group which showed its maladjustment through the lack of coordination of the two independent dimensions, breaking the assumed "non-rejection and acceptance" continuum. This group was made up of the third conglomerate which obtained a very high level of non-rejection 
$(\mathrm{M}=4.2 ; \mathrm{SD}=0.393)$ but at the same time a very low level of acceptance $(\mathrm{M}=1.68 ; \mathrm{SD}=0.386)$, which we have referred to as a group of "partial tolerance". The box-and-whisker diagram below expresses this graphically (Figure 5).

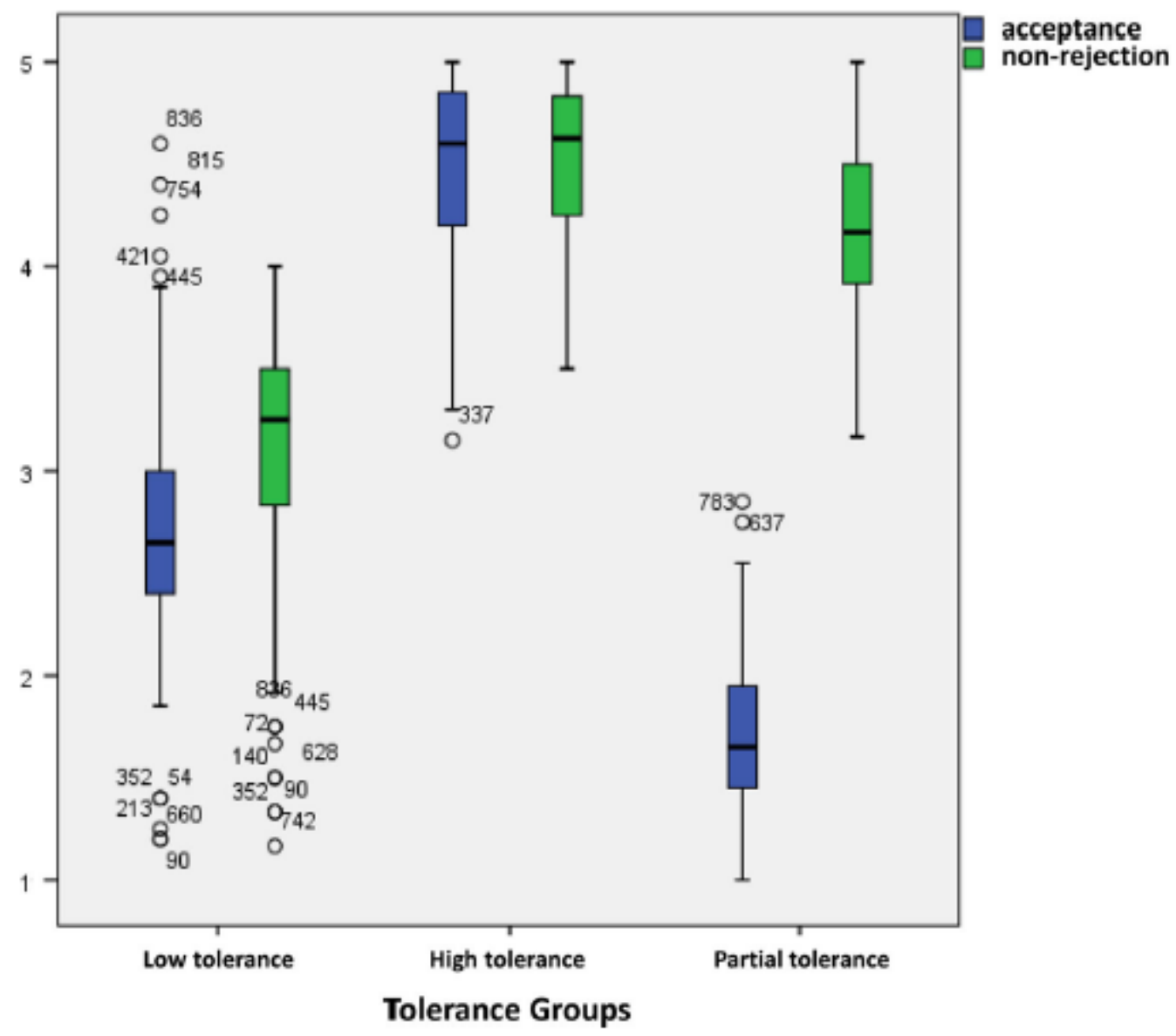

Fig. 5 Profiles of tolerance of sexual diversity in sport

To sum up, we identified three clearly differentiated profiles, with each of them accounting for roughly one third of the number of cases in the total sample (Table 3).

Table 3.

Cases in each group or profile of tolerance. Own source.

\begin{tabular}{llll}
\hline \multirow{3}{*}{ Conglomerates } & 1. Low tolerance & 251 & $32.7 \%$ \\
& 2. High tolerance & 276 & $36.0 \%$ \\
& 3. Partial tolerance & 240 & $31.3 \%$ \\
\hline Valid cases & & 767 & $87.3 \%$ \\
Lost cases & & 112 & $12.7 \%$ \\
Total & & 879 & \\
\hline
\end{tabular}


Once we had managed to determine the presence of these three modalities of tolerance and the cases associated to each one, we were then able to look at the cultural origin of each profile, and whether they were associated to the Spanish or UK subsamples.

\section{Identification and Description of the Distribution of Profiles According to Country}

The association of the tolerance profiles to the two cultural contexts was very high. Surprisingly, we obtained extremely high association coefficients $(\mathrm{Phi}=.908 / \mathrm{CC}=.672 ; \mathrm{p}=.000)$ stemming from the unequal presence of the totality of the partial tolerance group within the Spanish subsample (100\%) together with the near total presence of the low tolerance group (85.3\%) in this sample, whereas the high tolerance group was almost specifically located in the UK sample (99.6\%).

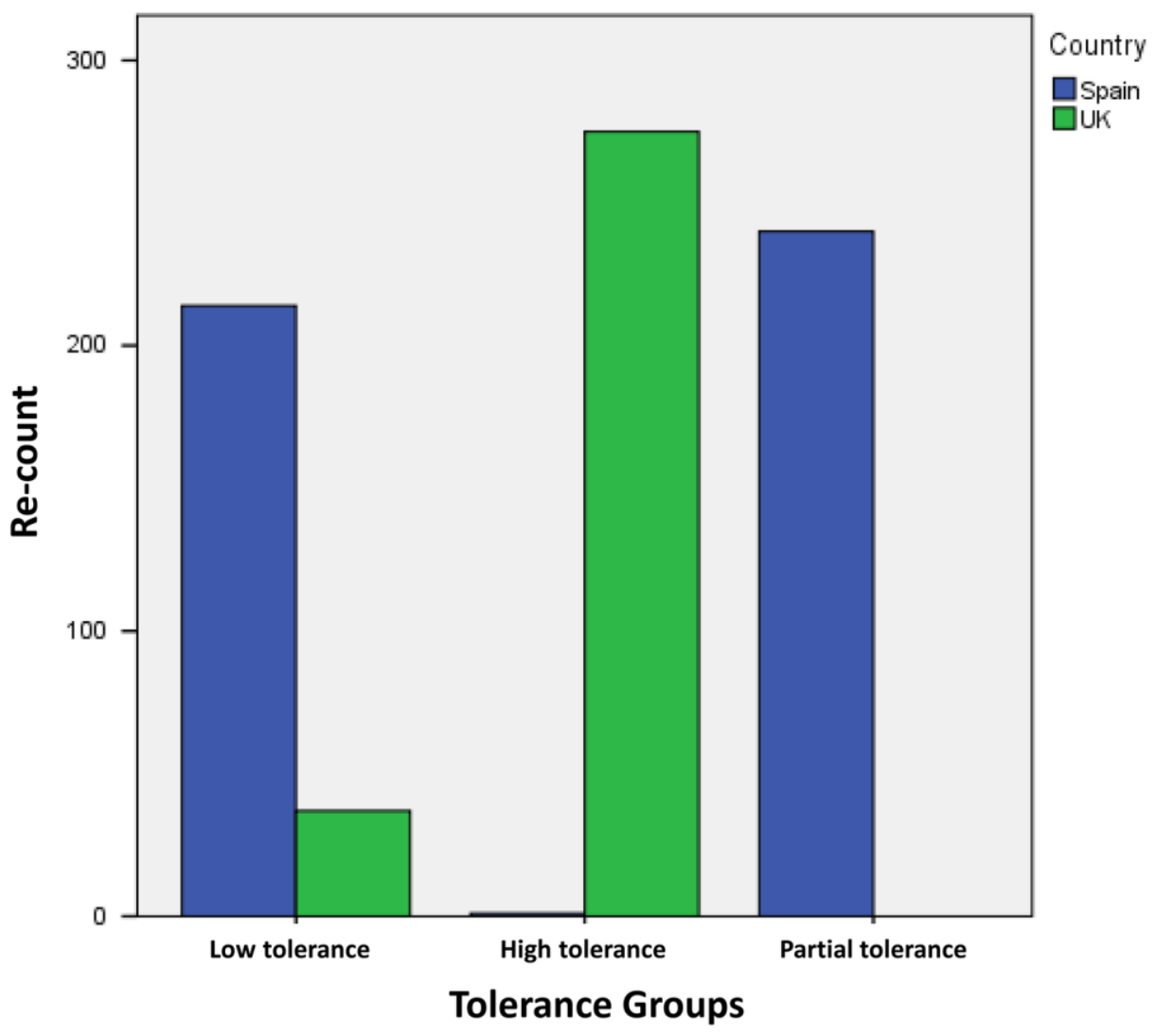

Fig. 6 Tolerance profiles per cultural contexts 
In short, the results illustrated a clear imbalance of data in favor of high tolerance in the United Kingdom. However, in the case of the sample from Spain, all the data indicated that the levels of tolerance along with the metacognitive profiles associated to the "non-rejection and acceptance" continuum which corresponded to them - were those which involved worse results, for their metacognitive maladjustment in one case (partial tolerance) or for the adjustment associated to intolerant attitudes (low tolerance). Finally, we should point out that the groups with worse prognosis in tolerance tended to be dominated by men (LowT $=83.5 \%$; PartialT $=55.6 \%)$, although there was clearly still a presence of women $($ LowT $=16.5 \%$; PartialT $=44.4 \%)$, so that these findings did not associate very strongly with sex $(\mathrm{CC}=.236 ; \mathrm{p}=.000)$.

\section{Discussion}

In line with recent studies, this paper underlines the importance of the analysis of tolerance towards sexual diversity in sport (Caudwell 2013; Griffin 2012; Walther 2006), because different positionings and attitudes linked to the presence in teams and sporting activities of people with non-heteronormative sexualities continue to be encountered. While many studies have pointed to improvements in the climate of tolerance within sport (Adams and Anderson 2012; Anderson, McCormack and Lee 2012; Channon and Matthews, 2015; Piedra 2015; Kauer and Krane 2006), it is also true, as is shown in our study, that there still exists a significant number of people who show lower levels of tolerance towards sexual diversity (Ensign et al. 2011; Oswalt and Vargas 2013).

Specifically, in the Spanish setting this coincides with the findings of Piedra (2015) which already discerned a metacognitive maladjustment in Spanish society regarding the elaboration of a superficial and politically correct discourse, which was not internally persuasive on an individual level. The difference observed between the more inclusive British context and the Spanish context where cultural homophobia persists, together with a certain level of homohysteria, positively demonstrates the existence of differentiated mental processes which other studies across multiple cultural contexts have not yet been able to directly show. This study expressly analyzes these differences in mental processing and identifies a key point of cognitive maladjustment, identifying a clear trend towards what we describe as a "pseudo-inclusive" climate.

Delving further into cultural differences, recent studies have tackled the progression of stages of homohysteria in Arab societies (Hamdi, Lachheb and Anderson 2016a; 2016b), identifying crucial elements of stages such as homoerasure and homohysteria. However, the case of Spanish culture does not seem to be on similar levels of development of tolerance towards sexual diversity (within sport), but nor is it at the stage of 
inclusivity as would seem to be the case of the UK; rather, Spain seems to exist within an intermediate stage of pseudo-inclusivity. In attempting to explain these observed divergences, the great cultural differences which exist between countries in this respect are most likely a consequence of their political, religious and social history, as emphasized in the recent study by Hamdi, Lachheb and Anderson (2016a; 2016b). In this sense it is possible that the Spanish sample's evident pseudo-inclusivity can be identified as a "politically correct" departure from the rejection of sexual diversity (and thus the negative implication of "being homophobic") alongside a concurrent refusal of acceptance of sexual minorities, shaped perhaps by the heteronormativity of Spain's traditionally conservative political and religious history.

What we describe here as pseudo-inclusivity has recently been alluded to by Anderson and McCormack (2016) in their paper on the refinement of inclusive masculinity theory; specifically, in responding to the critique that the perspective overstates the decline of homophobia, they note that "homophobia can decrease at the same time as heteronormativity and heterosexism persist” (p. 5). Borrowing Ghaziani’s (2014) notion of 'performative progressiveness', they identify a tendency for anti-homophobic discourse to prevail among groups of heterosexual people who do not necessarily "practice (anti-homophobic ideals) in their lives" (p.5). This observation is an important corrective to what we believe to be an often heavy-handed and unfair critique of inclusive masculinity theory - that it is based on an optimistic misrecognition of decreasing homophobia, and that it overstates the positive changes that have occurred in societies that have become less (overtly) homophobic. The data from our study provide new empirical insight which is useful in the context of this debate, revealing the manifestation of pseudo-inclusivity through statistically significant evidence of a metacognitive profile involving the simultaneous non-rejection and non-acceptance of sexual minority groups. In this sense, our data support the more nuanced appreciation of the dynamics of social change framed by recent refinements of inclusive masculinity theory, showing that heteronormativity can coexist with decreasing homophobia. This allows us to better understand on-going negativity in attitudes towards sexual diversity without calling into question the central contention that overt homophobia has significantly declined in (most) Western societies over recent decades, opening up wider scope for analyses of how such changes affect the lives of sexual minority groups.

Thus, we believe that a specific contribution of this study in relation to previous offerings has been to identify the characteristics of an intermediate state of pseudo-inclusivity, characterized by what we have called partial tolerance towards sexual diversity, which is distinct from full acceptance and inclusion. This original contribution comes from having considered the construct of tolerance as composed of the bi-dimensionality of 
non-rejection and acceptance - constructs which have previously been implicitly positioned as continuous but in fact exist independently of each other, and should be handled as such in future investigations of this phenomenon. As such, this research confirms existing studies in the UK, updates empirical work on Spain, and provides a tool for more accurate quantitative and cross-cultural examinations in these and other countries, in line with McCormack and Anderson's (2014, p.117) call to interrogate "shifting homophobias in a global context".

\section{Conclusions}

Policies adopted to reduce or wipe out homophobia in sport help to prevent discrimination and show great advances towards inclusion (Waldron 2015). These strategies help to make the oppressed more visible and create a more inclusive environment. In this process of social change, ideas have been formulated about the stages in the development and implementation of real social inclusion based on tolerance towards diversity. Drawing on the proposal of Anderson (2009; see also Anderson et al. 2016) regarding three social stages (homoerasure, homohysteria, and inclusivity), we have enlarged the theory by adding the "pseudo-inclusive" stage which operates in circumstances of incomplete social inclusion of diversity, and tolerance towards this in sporting spheres. This is confirmed through the study of the adjustment and capacity of metacognitive regulation of relational decision-making, showing a clear correspondence between this stage and Spanish society (Piedra 2015), in contrast to that for UK society where tolerance and inclusivity appear to be more deeply rooted.

Indeed, a line of interest for research facilitated by the instrument validated here is the possibility of applying the same logic to other cultural fields and settings in Spain, not necessarily sport-related, to determine the extent and impact of policies affecting citizenship regarding social pedagogy applied to the development of attitudes of tolerance towards sexual diversity. The results of this study show clearly that this social pedagogy has not reached totally as far as Spanish men and women in the world of sport are concerned. Although progress has clearly been made in terms of tolerance, it would seem that these ideas have not taken root in ways which prompt full validation of sexual minority groups. It also seems worthwhile to analyze the differences which may arise between the strategies developed to reduce homophobia/promote inclusivity in the UK setting, compared to those applied in Spain, to try to better explain the difference which is manifested in this empirical study.

As in all research, this study has a series of limitations which may, to a certain extent, affect the result due to the methodological procedures and theoretical frameworks applied. Perhaps the most significant limitation of the study is that its design seemed to replicate the tendency to imagine homophobic/heterosexist 
attitudes in one-dimensional terms, by not suitably distinguishing between various different forms of prejudice facing diverse sexual minority groups, such as biphobia and transphobia (see Worthen 2013). The data gathered did not suggest particular differences in these constructs, although it is possible - if not likely - that this is because the initial design of the instrument did not seek to overtly foreground them as elements of analysis, rather than the existence of no actual differences in participants' attitudes.

In addition, our use of inclusive masculinity theory, a perspective emerging predominantly from research on gay men, similarly shapes the conclusions we have developed. This is not to say that our observation regarding 'partial tolerance' cannot be instructive in various ways for scholarship on all forms of sexual diversity, but rather to stress that its conceptual roots lie in a theory which specifically pertains to changes in the relationship between men, homophobia and masculinity (Anderson \& McCormack, 2016). As such, future efforts at evaluating differing levels of inclusivity may benefit from specifically differentiating between forms of prejudice experienced by different sexual minority groups, if not also mapping them against varied gender and sexuality characteristics and different theoretical frames, in order to measure the varied manifestations of (in)tolerance among and towards diverse groups of (sports)people.

Another drawback is that although a correlational design using a questionnaire can cater easily for a larger sample size, as used in this study, it may not be the best design for asking participants about their perception of socially controversial subjects such as that which we explored. Indeed, there are more powerful designs (for example, of a qualitative type) that could be employed in future studies of this phenomenon to paint more detailed pictures of current attitudinal norms within these and other cultural spaces. Finally, the use of different procedures for the British and Spanish samples (online/pencil and paper, respectively) may have affected the sample characteristics of the two countries, which needs bearing in mind regarding the representativeness of the results. However, despite these limitations, we believe we have made an original contribution with respect to our results and measurement methodology, which may be confirmed by, or be of use in, similar future studies.

\section{Ethical considerations}

\section{Conflict of interest}

The authors declare that they have no conflict of interest.

\section{Ethical Approval}


At all times, we respected the ethical principles of the Belmont Report: the principle of beneficence, the principle of respect for human dignity and the principle of justice. In addition, the study was approved by the regional ethical review board of the lead author's university prior to commencing data gathering.

\section{Informed Consent}

All participants received a short information sheet at the front of the paper or online surveys, which explained the overarching aims of the project and specified participants' right to withdraw at any time. Participants gave their consent to participate voluntarily by proceeding past the first page of the online survey. All responses were anonymized; no identifying information was gathered about participants or the clubs they were affiliated to.

\section{References}

Act 3/2007 (2007). Ley de Igualdad Efectiva de mujeres y hombres [Effective equality of women and men Act]. Retrieved from http://www.boe.es/boe/dias/2007/03/23/pdfs/A12611-12645.pdf.

Act 19/2007 (2007). Ley contra la violencia, el racismo, la xenofobia y la intolerancia en el deporte [Against violence, racism, xenophobia, and intolerance in sports Act]. Retrieved from https://www.boe.es/boe/dias/2007/07/12/pdfs/A29946-29964.pdf

Adams, A., \& Anderson, E. (2012). Exploring the relationship between homosexuality and sport among the teammates of a small Midwestern Catholic College soccer team. Sport, Education and Society, 17(3), 347363. doi:10.1080/13573322.2011.608938

Anderson, E. (2009). Inclusive masculinity: The changing nature of masculinities. New York, NY: Routledge.

Anderson, E., Magrath, R., \& Bullingham, R. (2016). Out in Sport. The experiences of openly gay and lesbian athletes in competitive sport. London, UK: Routledge.

Anderson, E. \& McCormack, M. (2016). Inclusive masculinity theory: Overview, reflection and refinement. Journal of Gender Studies, Online First, 1-15. doi:10.1080/09589236.2016.1245605

Anderson, E., McCormack, M., \& Lee, H. (2012). Male team sport hazing initiations in a culture of decreasing homohysteria. Journal of Adolescent Research, 27, 427-448. doi:10.1177/0743558411412957

Barber, H., \& Krane, V. (2007). Creating inclusive and positive climates in girls' and women’s sport: Position statement on homophobia, homonegativism, and heterosexism. Women in Sport \& Physical Activity Journal, 16(1), 53-56. 
Barbero, J. I. (2003). La educación física y el deporte como dispositivos normalizadores de la heterosexualidad.

[Physical education and sports as normalizing mechanisms of heterosexuality]. In O. Guasch, \& O.

Viñuales (eds.), Sexualidades: Diversidad y control social (pp. 355-377). Barcelona, Spain: Bellaterra.

Caudwell, J. (2013). 'Does your boyfriend know you're here?' The spatiality of homophobia in men's football culture in the UK. In J. Caudwell, \& K. Browne (eds.), Sexualities, Spaces and Leisure Studies (p. 9-24). London, UK: Routledge.

Caudwell, J. (2014). [Transgender] young men: Gendered subjetivities and the physically active body. Sport, Education and Society, 19(4), 398-414.

Channon, A., \& Matthews, C. R. (2015). 'It is what it is': Masculinity, homosexuality, and inclusive discourse in mixed martial arts. Journal of Homosexuality, 62(7), 936-956. doi:10.1080/00918369.2015.1008280

Chin, M. M., \& Kuo, S. W. (2010). From metacognition to social metacognition: Similarities, differences, and learning. Journal of Education Research, 3(4), 321-338.

Cleland, J. (2013). Discussing homosexuality on association football fan message boards: A changing cultural context. International Review for the Sociology of Sport, 50(2) 125-140.

Connell, R. (1995). Masculinities. Los Angeles, CA: University of California Press.

Davis-Delano, L. R. (2014). Sport as context for the development of women's same-sex relationships. Journal of Sport and Social Issues, 38(3), 263-285. doi:10.1177/0193723513520554

Ensign, K., Yiamouyiannis, A., White, K., \& Ridpath, D. (2011). Athletic trainers' attitudes toward lesbian, gay, and bisexual National Collegiate Athletic Association student-athletes. Journal of Athletic Training, 46(1), 69-75. doi: 10.4085/1062-6050-46.1.69.

Gill, D. L., Morrow, R. G., Collins, K. E., Lucey, A. B., \& Scultz, A. M. (2010). Perceived climate in physical activity settings. Journal of Homosexuality, 57(7), 895-913. doi:10.1080/00918369.2010.493431.

Griffin, P. (2012). LGBT equality in sports: Celebrating our successes and facing our challenges. In G. B. Cunningham (Ed.), Sexual orientation and gender identity in sport: Essays from activists, coaches, and scholars (pp. 1-12). College Station, TX: Center for Sport Management Research and Education.

Hamdi, N., Lachheb, M., \& Anderson, E. (2016a). Queen of fights: Lesbians in Tunisian sports. Journal of Homosexuality, 63(8), 1127-1145. doi: 10.1080/00918369.2015.1117902

Hamdi, N., Lachheb, M., \& Anderson, E. (2016b). Masculinity, homosexuality and sport in an Islamic state of increasing homohisteria. Journal of Gender Studies, Online First, 1-14. doi:

$10.1080 / 09589236.2016 .1155979$ 
Herek, G. M. (1984). Attitudes toward lesbians and gay men: A factor analytic study. Journal of Homosexuality, $10(1 / 2), 39-51$.

Herek, G. M. (1988). Heterosexuals' attitudes toward lesbians and gay men: Correlates and gender differences. Journal of Sex Research, 25, 451-477.

Herek, G. M., \& Gonzalez-Rivera, M. (2006). Attitudes toward homosexuality among U.S. residents of Mexican descent. Journal of Sex Research, 43, 122-135.

Kauer, K., \& Krane, V. (2006). “Scary dykes” and "Feminine Queens": Stereotypes and female collegiate athletes. Women in Sport \& Physical Activity Journal, 15(1), 42-55

Krane, V., \& Barber, H. (2003). Lesbian experiences in sport: A social identity perspective. Quest, 55, 328-346. doi: $10.1080 / 00336297.2003 .10491808$

Martí, E. (1995). Metacognición: Entre la fascinación y el desencanto. [Metacognition: Between fascination and disillusion]. Infancia y Aprendizaje, 18(72), 9-32.

McCormack, M., \& Anderson, E. (2010). "It's just not acceptable any more": The erosion of homophobia and the softening of masculinity at an English sixth form. Sociology, 44(5), 843-859.

McCormack, M., \& Anderson, E. (2014). The influence of declining homophobia on men's gender in the United States: An argument for the study of homohysteria. Sex Roles, 71(3), 109-120.

Morrow, R. G., \& Gill, D. L. (2003). Perceptions of homophobia and heterosexism in physical education. Research Quarterly for Exercise and Sport, 74(2), 205-214.

O’Brien, K. S., Shovelton, H., \& Latner, J. D. (2013). Homophobia in physical education and sport: The role of physical/sporting identity and attributes, authoritarian aggression, and social dominance orientation. International Journal of Psychology, 48(5), 891-899. doi: 10.1080/00207594.2012.713107.

Oswalt, S. B., \& Vargas, T. M. (2013). How safe is the playing field? Collegiate coaches' attitudes towards gay, lesbian, and bisexual individuals. Sport in Society, 16(1), 120-132. doi: 10.1080/17430437.2012.690407

Piedra, J. (2015). Gays y lesbianas en el deporte: Discurso de jóvenes universitarios españoles en torno a su aceptación. [Gays and lesbians in sport: University students' speech about their acceptance]. Movimento, 21(4), 1067-1081.

Piedra, J. (2016). Escala de actitudes hacia la diversidad sexual en el deporte (EDSD): desarrollo y validación preliminar. [Attitude scale towards sexual diversity in sport: development and preliminary validation]. Revista de Psicología del Deporte, 25(2), 299-307. 
Piedra, J., Rodríguez-Sánchez, A. R., Ries, F., \& Ramírez-Macías, G. (2013). Homofobia, heterosexismo y educación física: Percepciones del alumnado. [Homophobia, heterosexism and physical education: Students' perceptions]. Profesorado. Revista de Currículum y Formación del Profesorado, 17(1), 325-338.

Pronger, B. (2000). Homosexuality and sport. Who’s winning? In J. McKay, M. A. Messner, \& D. Sabo (Eds.), Masculinities, Gender Relations, and Sport (pp. 222-244). London, UK: SAGE.

Waldron, J. J. (2016). It's complicated: Negotiations and complexities of being a lesbian in sport. Sex Roles, 74(7), 335-346. doi:10.1007/s11199-015-0521-x

Walther, T. (2006). Kick it out. Homophobia in football. Amsterdam, NL: European Gay and Lesbian Sport Federation.

Worthen, M. G. F. (2013). An argument for separate analyses of attitudes toward lesbian, gay, bisexual men, bisexual women, MtF and FtM transgender individuals. Sex Roles, 68(11), 703-723. doi: 10.1007/s11199012-0155-1 